\title{
Immediate Neural Plasticity Shapes Motor Performance
}

\author{
Michael C. Dorris, Martin Paré, and Douglas P. Munoz \\ Medical Research Council Group in Sensory-Motor Neuroscience, Department of Physiology, Queen's University, \\ Kingston, Ontario, Canada K7L 3N6
}

\begin{abstract}
The consolidation of motor skills necessitates long-lasting changes in the nervous system. For the most part, plasticity has been documented in motor systems after training and longterm adaptation. However, there has been no demonstration of immediate neural changes associated with the rapid adaptation of motor behavior required to interact with a dynamic environment. To address this issue, we explored the changes in performance (reaction time) of rhesus monkeys that executed saccadic eye movements to one of two visual stimuli while monitoring the preparatory activity of neurons in the superior colliculus, a structure close to the motor output. Similar to the well established sequential effects observed in human manual responses, each monkey displayed reaction times to target
\end{abstract}

locations that were organized in a sequential pattern, becoming progressively shorter with each preceding repeated movement and longer with each preceding nonrepeated movement. This sequential pattern of performance modification was associated with concordant changes in the preparatory activity of superior colliculus neurons in advance of the saccadic target presentation. These data indicate that neural properties are continuously shaped by use-related experience in a manner consistent with the progressive adaptation of motor behavior.

Key words: superior colliculus; saccade; reaction time; motor preparation; motor learning; sequential effects; repetition effect; oculomotor; gap paradigm; monkey
It is well established that extensive training in a stimulus-response task leads to adaptive changes in the performance of the required behavior (Anderson, 1980; Schmidt, 1991). For example, in the oculomotor system, behavioral training can invoke changes in the metrics of eye movements (McLaughlin, 1967; Miles and Eighmy, 1980) and their reaction times (Fischer and Ramsperger, 1986; Paré and Munoz, 1996). The changes in the activity and the connections of neuronal populations that underlie experiencerelated modifications in behavior have been termed neural plasticity (Miles and Lisberger, 1981; Buonomano and Merzenich, 1998). Because these modifications in the quality of motor responses can be relatively time-consuming, the examination of ongoing plasticity in neuronal ensembles is technically difficult. As a consequence, previous experiments have, for the most part, only documented physiological differences before and after training (Pascual-Leone et al., 1994; Karni et al., 1995; Nudo et al., 1996; Classen et al., 1998). Only recently have studies begun to probe the time course of neural plasticity by tracking the evolution of behavior and neural changes within an experimental session (Mitz et al., 1991; Dorris and Munoz, 1998; Nakamura et al., 1998). However, changes to the nervous system that occur with a time scale of hours or minutes will not suffice to interact with a dynamic environment. Instead, organisms must continuously modulate their nervous systems. This on-line process was recognized by Mountcastle (1995), who suggested that "the most

Received July 14, 1999; revised Oct. 11, 1999; accepted Oct. 28, 1999.

This work was supported by a group grant from the Medical Research Council of Canada. M.C.D. was supported by a Queen's University graduate fellowship and an Ontario Graduate Science and Technology scholarship. D.P.M. is a Medical Research Council Scientist. We thank A. Lablans and D. Hamburger for technical assistance and B. Corneil, S. Everling, D. P. Hanes, G. Loeb, P. K. Rose, and S. H. Scott for commenting on an earlier version of this manuscript.

Correspondence should be addressed to Douglas P. Munoz, Department of Physiology, Queen's University, Kingston, Ontario, Canada K7L 3N6. E-mail: doug@eyeml.queensu.ca.

Copyright (C) 1999 Society for Neuroscience $\quad 0270-6474 / 99 / 200001-05 \$ 15.00 / 0$ powerful and obvious plasticity is the regulation and control of dynamic activity ... over short time scales by the afferent modulatory systems of the central core, producing adaptive changes in an otherwise unchanging connectivity. Here is plasticity a 'gogo'." Our goal was to elucidate a neural correlate of this rapid plasticity by using a novel approach, which consisted of studying ongoing changes in neuronal activity associated with immediate changes in motor performance that occurred during a saccadic eye movement task wherein the movement's goal was constantly changing.

\section{MATERIALS AND METHODS}

Subjects. Three male rhesus monkeys (Macaca mulatta) weighing between 6 and $9 \mathrm{~kg}$ were used for this study. We used standard techniques to record the extracellular activity of single neurons in the intermediate layers of the superior colliculus (SC), measure eye movements, and convolve spike trains with a postsynaptic activation function as described previously (Dorris and Munoz, 1998). All procedures were approved by the Queen's University Animal Care Committee and complied with the guidelines of the Canadian Council on Animal Care.

Task procedures. The monkeys were trained to perform the gap saccade paradigm as described previously (Dorris et al., 1997). The gap saccade paradigm was used because the removal of the fixation point facilitates the preparation of the upcoming saccade by releasing active fixation (Dias and Bruce, 1994; Dorris and Munoz, 1995), and it also acts as a

This article is published in The Journal of Neuroscience, Rapid Communications Section, which publishes brief, peerreviewed papers online, not in print. Rapid Communications are posted online approximately one month earlier than they would appear if printed. They are listed in the Table of Contents of the next open issue of JNeurosci. Cite this article as: JNeurosci, 2000, 20:RC52 (1-5). The publication date is the date of posting online at www.jneurosci.org.

http://www.jneurosci.org/cgi/content/full/3837 
warning cue (Ross and Ross, 1980). Furthermore, the gap saccade paradigm disinhibits saccade-related neurons in the SC, facilitating preparatory neuronal activity before target presentation (Dorris et al., 1997; Dorris and Munoz, 1998). Each trial was preceded by a $1000 \mathrm{msec}$ intertrial interval. The trial started with the monkey fixating a central fixation point (back-projected light-emitting diode, $2.0 \mathrm{~cd} / \mathrm{m}^{2}$ ) for $500-$ $1000 \mathrm{msec}$. The fixation point was extinguished, and there was a $200 \mathrm{msec}$ gap period before an eccentric target (also $2.0 \mathrm{~cd} / \mathrm{m}^{2}$ ) appeared. We restricted our analysis to trials in which the saccades ended within $3^{\circ}$ of the final saccadic target and with saccadic reaction times (SRTs) between 70 and $300 \mathrm{msec}$. For each neuron, a block of 50-100 trials was performed in which the target location was pseudorandomized between the center of the response field of the neuron and a position equidistant relative to the fixation point but in the diametrically opposite direction.

Neuron classification. While the monkey performed the gap saccade paradigm, we recorded and subsequently analyzed the discharge of a subset of saccade-related neurons located within the SC intermediate layers (depth, 1.0-3.0 mm) with (1) saccade-related activity above 100 spikes/sec for saccades into the center of the response field of the neuron and (2) early, pretarget activity during the end of the gap period (50 msec before to $50 \mathrm{msec}$ after target onset) that was significantly greater than during visual fixation (the $100 \mathrm{msec}$ preceding FP disappearance; paired $t$-test, $p<0.05)$.

Data analysis. Sequential effects have been characterized extensively behaviorally using both saccadic (Jüttner and Wolf, 1992) and manual responses (Bertelson, 1961; Soetens, 1998). For all sequential analyses of our data, only those sequences with successive correct trials were included. For the SRT analysis (i.e., Figs. 1, 3C,D), we refer to compatible as the condition in which the previous saccade was of the same metric as the current saccade and incompatible as the condition in which the previous saccade was of the opposite metric as the current saccade. This analysis was performed separately for both monkeys and for saccades directed into the left and right hemifields. Similar sequential patterns were observed in all cases, which allowed us to collapse the data across saccade direction and monkeys.

Compared with the equivalent for SRT, compatible and incompatible have slightly different definitions for the analysis of neuronal activity (i.e., Figs. 2, 3A,B, 4). As we discuss below, neuronal activity was sampled during an epoch before any target information reaches these SC neurons. Therefore, neuronal activity during this pretarget epoch did not differ with the direction of the impending target $(t$ test, $p>0.05)$. For neuronal activity, we refer to compatible as the condition in which the previous saccade was directed into the response field of the neuron, irrespective of the direction of the saccade on the current trial. Conversely, we refer to incompatible as the condition in which the previous saccade was directed opposite the response field of the neuron, irrespective of the direction of the saccade on the current trial. We present the data using the slightly different terms of compatible and incompatible for neuronal activity and SRT as such for ease of presentation. We also performed all sequential analyses according to current saccade direction and corresponding neuronal activity (i.e., four "N-1" conditions: SRT compatible/response field compatible, SRT compatible/response field incompatible, SRT incompatible/response field compatible, and SRT incompatible/response field incompatible), and all statistically significant comparisons reported below were maintained.

We estimated the preparatory neuronal activity from a postsynaptic spike activation function (Hanes and Schall, 1996) on a trial-by-trial basis during an epoch lasting from 40 to $50 \mathrm{msec}$ after target presentation. We labeled this epoch "pretarget" because it represents the neuronal firing rate just before any change that could be induced by visual inputs caused by the presentation of the peripheral target. We used a Poisson spike train analysis (Hanes et al., 1995) to determine the onset of the visually aligned burst for each neuron, and we performed a running $t$ test between the average compatible and incompatible waveforms for each neuron. We found no evidence for the influence of the visual stimulus on neuronal discharge until $>60 \mathrm{msec}$ after target presentation with either method.

The percent change in both neuronal and SRT between the N-1 compatible and incompatible conditions (see Fig. $3 B, D$ ) was calculated as follows: change in neuronal activity $=[$ (compatible neuronal activity incompatible neuronal activity $) /($ compatible neuronal activity + incompatible neuronal activity)/2) ${ }^{*} 100 \%$; and change in SRT $=[$ (compatible SRT - incompatible SRT $) /[(($ compatible SRT + incompatible SRT $) /$ 2) -80$] * 100 \%$.

The $80 \mathrm{msec}$ constant was subtracted when calculating the change in SRT because it represents the amount of the SRT that is fixed because of

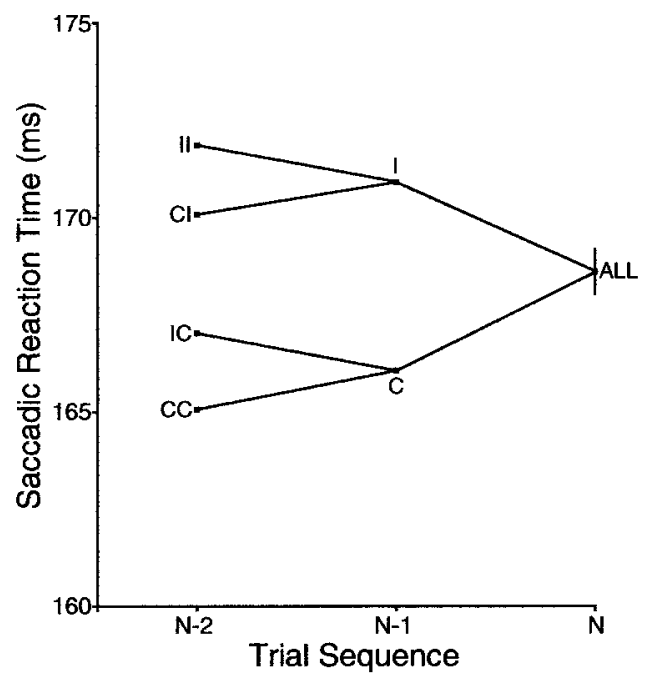

Figure 1. Distribution of mean SRTs as a function of the sequence of previous saccades. Extensive behavioral data from two of the monkeys (monkey 1, 1762 saccades; monkey 2, 1560 saccades) showed the same pattern and were combined. The current SRT is shown irrespective of the previous saccades $(N)$ or based on the compatibility of the metrics of the previous saccade $(N-1)$ or previous two saccades $(N-2)$ with the current saccade. The compatibility of recent to distant saccades is determined by reading from right to left with each saccade being either compatible $(C)$ or incompatible $(I)$ with the saccade on the current trial. Both saccade locations $\left(10^{\circ}\right.$ right and $10^{\circ}$ left $)$ were combined. The SEM is shown for the $A L L$ condition.

afferent and efferent processes (Carpenter, 1981) and therefore cannot be affected by changes in the neuronal activity of the SC neurons recorded here.

\section{RESULTS}

To assess any immediate changes in motor performance, we measured the variation in SRT contingent on the history of preceding saccades. We used a gap saccade paradigm wherein monkeys made a saccade from a central visual stimulus to an eccentric target that appeared at one of two diametrically opposite locations with equal probability. Even in such a simple task, SRTs display a large variability that traditionally has been hypothesized to be stochastic in nature (Carpenter, 1981). However, when the sequence of previous saccades is taken into account, it becomes apparent that the different lineages of saccades that comprise the SRT distribution form a sequential pattern (Fig. 1). SRTs decreased with each preceding target-directed saccade with compatible metrics and increased with each preceding targetdirected saccade with incompatible metrics. These sequential effects are one factor contributing to the variability in SRT distributions observed under stable experimental conditions. We hypothesized that this sequential pattern of behavior must be shaped by underlying neural plasticity that, like the behavior, is immediate, progressive, and reversible.

With a microelectrode located in the intermediate layers of the superior colliculus, a structure close to the motor output, we recorded from saccade-related neurons that increased their activities during the gap period in a manner consistent with a role in saccade preparation (Dorris et al., 1997; Dorris and Munoz, 1998). As exemplified in Figure $2 A$, the activity of these neurons increased during the gap period, irrespective of the target location. Shortly after target presentation, either these neurons produced a high-frequency burst of action potentials for saccades compatible with the response field of the neuron, or their activity 


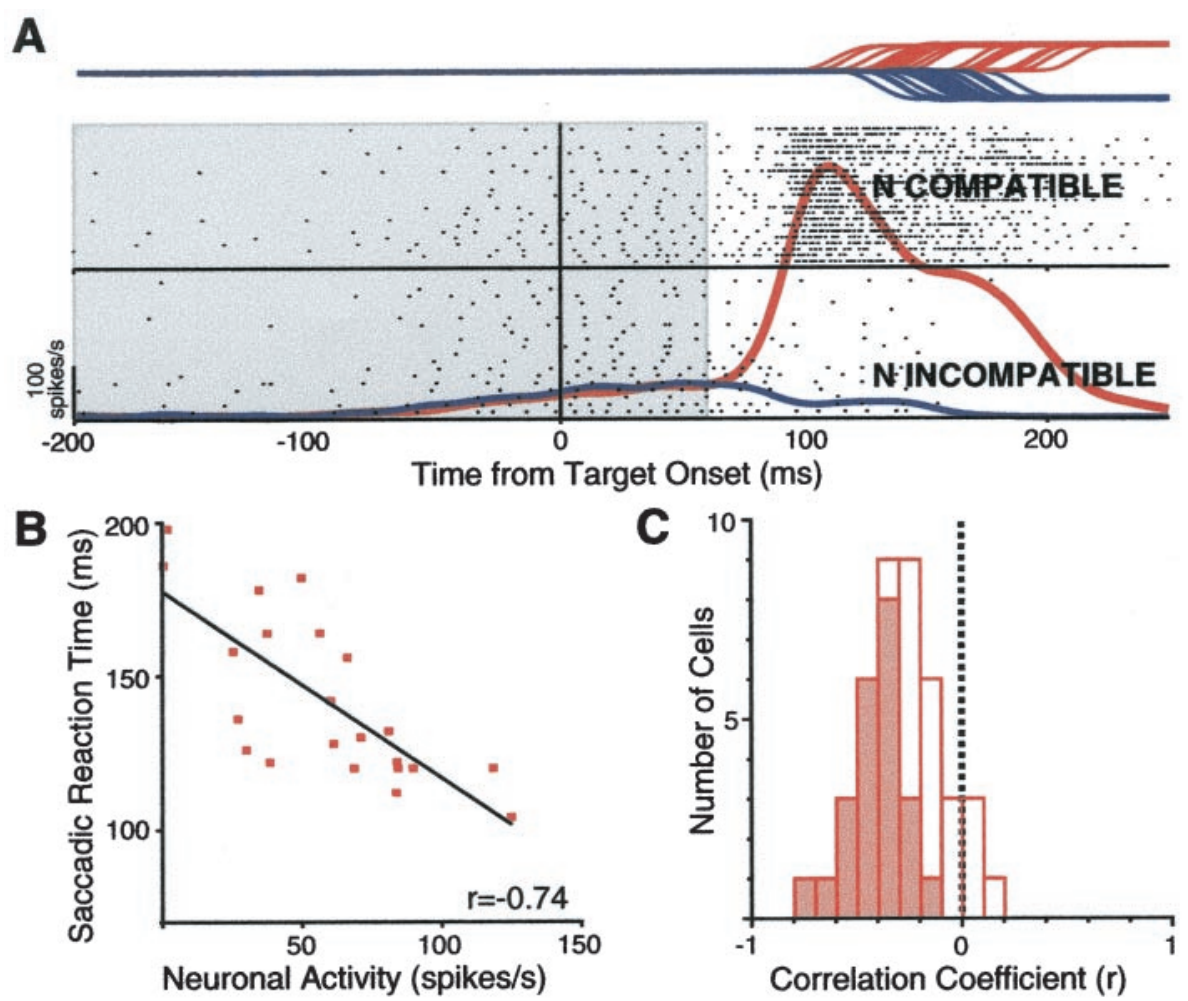

D

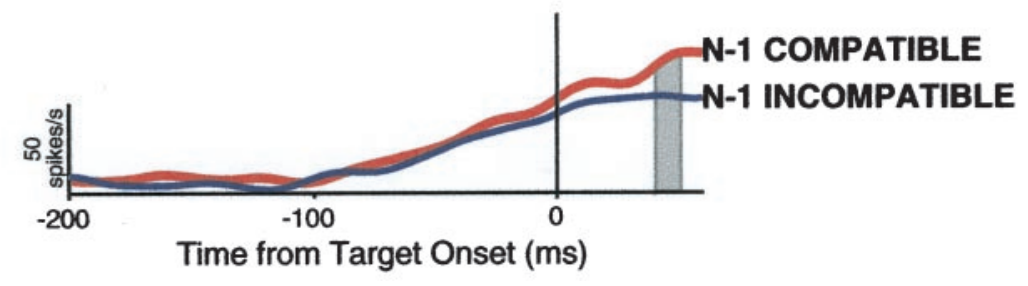

Figure 2. Pretarget neuronal activity predicts future performance and is shaped by past experience. $A$, Each tick mark in the rasters represents an individual action potential, and each horizontal line of rasters represents the neuronal activity during one trial. Horizontal eye position traces are shown in the top panel with up (red traces) denoting saccades compatible with the response field of the neuron and down (blue traces) denoting saccades incompatible with the response field of the neuron. Rasters, spike activation functions, and eye movement traces are aligned on target appearance (vertical line at time 0 ). $B$, Trial-bytrial pretarget activity of this single neuron plotted against SRT for compatible trials. $C$, The majority of neurons (38 of 42) had a negative correlation between neuronal activity and SRT on a trial-by-trial basis. The shaded region of the histogram shows the proportion of individual neurons with statistically significant correlations. $D$, Pretarget activity from $A$ is segregated based on whether the previous saccade $(N-1)$ was compatible (thick red line) or incompatible (thin blue line) with the response field of the neuron. The shaded region 40-50 msec after target appearance represents the epoch in which the neuronal activity was sampled for subsequent analysis. was suppressed for saccades incompatible with the response field of the neuron. In accordance with our previous studies, the level of pretarget preparatory activity of these collicular neurons predicted SRT when saccades were directed into their response field: the higher the pretarget activity on a trial, the shorter the ensuing SRT (Fig. 2B) (Dorris et al., 1997; Dorris and Munoz, 1998). For the sample of neurons $(n=42)$, the trial-by-trial correlation between the pretarget discharge rate for saccades compatible with the response field of the neuron and the ensuing SRT had a mean correlation coefficient of -0.28 , which differs significantly from zero ( $t$ test, $p<0.0001$; Fig. $2 C$ ). A little more than half of the individual neurons (22 of 42, 52\%) had statistically significant correlations (Fisher's $r$ to $z$ test, $p<0.05$ ).

The pretarget activity (Fig. $2 A$, shaded area) of a single neuron was indistinguishable regardless of whether the target of the current trial $(\mathrm{N})$ was presented compatible or incompatible with the response field of the neuron, because at this time there was no information regarding the upcoming target location. However, when the trials were segregated based on whether the metric of the previous (N-1) saccade was compatible or incompatible with the response field of the neuron (Fig. 2D), the pretarget activity was greater for trials preceded by compatible saccades. This was true even though the metric of the previous saccade (or target location) offered no probabilistic information regarding the location of the upcoming saccade in the current trial. In other words, half of the trials that comprise both the N-1 compatible and incompatible waveforms in Figure $2 D$ will be directed toward the response field of the neuron, and the other half will be directed opposite the response field of the neuron. Thus, the same neuronal activity both predicted future behavior (Fig. $2 B, C$ ) and reflected the past experience (Fig. 2D).

The pretarget neuronal activity of most neurons recorded in this study (81\%) was larger when the previous saccade metrics were compatible with the response field of each neuron (average, 52 spikes/sec) compared with incompatible metrics (45 spikes/sec) (paired $t$ test, $p<0.0001$; Fig. $3 A$ ). The change in neuronal activity between the segregated $\mathrm{N}-1$ compatible and incompatible trials averaged $18.4 \%$ (range, -20 to $80 \%$ ) for the population of neurons (Fig. $3 B$ ). During the experiments in which these same neurons were recorded, we also observed a sequential effect on the SRT of the corresponding saccades (Fig. 3C). In the majority of experiments $(86 \%)$, the SRT for trials with a previously compatible metric $(158 \mathrm{msec})$ was shorter than for those preceded by an incompatible metric $(166 \mathrm{msec})$ (paired $t$ test, $p<0.0001)$. The change in behavioral performance between incompatible and compatible trials covaried with the increase in neuronal activity and averaged $-10.0 \%$ (range, -37.5 to $7.0 \%$ ) (Fig. $3 D$ ). We also performed the same analysis on other behavioral measures. When saccadic velocity (paired $t$ test, $p=0.49$ ) and metrics (paired $t$ test, $p=0.49$ ) were tested, no sequential effects were found (data not shown).

Further concordance between neuronal activity and SRT alter- 
Figure 3. Effects of the direction of the previous saccade $(N-1)$ on pretarget neuronal activity and behavior for each block of trials in which a neuron was recorded $(n=42)$. $A$, Each data point represents the mean pretarget neuronal activity in the epoch $40-50 \mathrm{msec}$ after target presentation for a single neuron based on whether the previous saccade was compatible or incompatible with the response field of the neuron. The equality line (slope $=1$ ) is shown. $C$, Each data point represents the mean SRT during the corresponding blocks in which the neurons were recorded in $A$ based on whether the previous saccade location was compatible or incompatible with the current saccade location. $B, D$, Histograms showing the distribution of percent changes in neuronal activity $(B)$ and SRT $(D)$ for the experiments in $A$ and $C$, respectively.
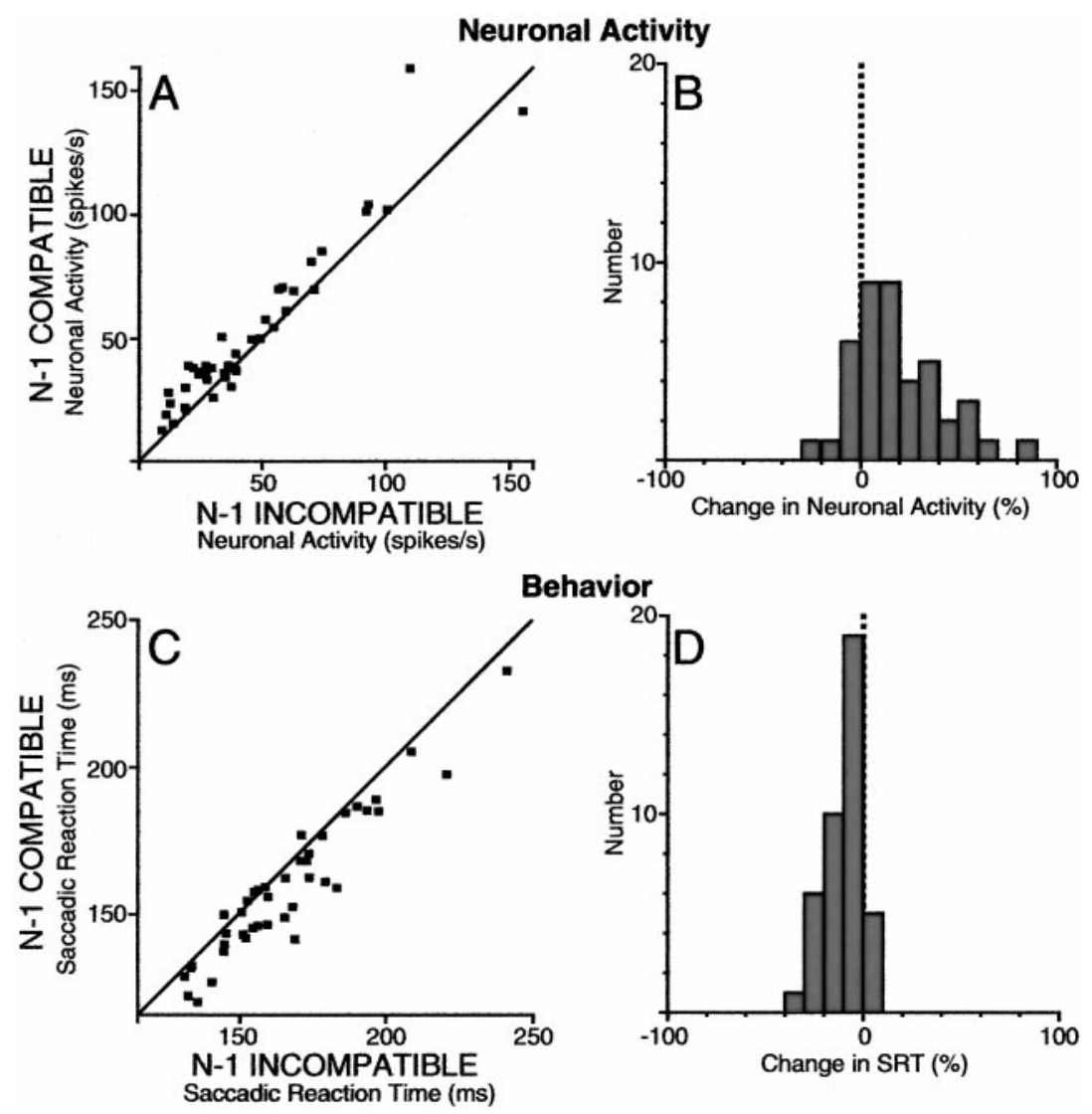

ations was manifest in the configuration of the sequential pattern of changes (compare Fig. 4 with Fig. 1); these two configurations were inverted with respect to each other because of the existing negative correlation between these two factors. The pretarget neuronal activity increased progressively with each preceding compatible saccade and decreased progressively with each preceding incompatible saccade. These sequential patterns of both behavioral and neuronal responses highlight the difficulty encountered when determining an objective baseline measurement of these variables. We argue that the neural processes underlying behavioral responses cannot be isolated in a single trial but must be recognized in the context of trial history.

\section{DISCUSSION}

In this study, we first unveiled a sequential pattern of monkey SRTs that depended on the history of preceding trial performance. Along with our previous finding that SRT depends on the state of readiness as estimated from the previous trial performance (Paré and Munoz, 1996), this is the first demonstration in monkey saccadic behavior of the sequential effects studied extensively in human manual tasks (Bertelson, 1961; Soetens, 1998). In addition, we demonstrated that this fast behavioral modification was associated with changes in preparatory neuronal activity that were immediate, progressive, and reversible. Thus, behavioral responses and the neural processes that control them are continuously shaped by previous experiences.

These findings provide further support that the oculomotor system is selectively alterable by use-related experience and demonstrate the rapidity in which natural behavioral conditions can induce changes in neural activity within motor systems. We put forth the possibility that these rapid neural changes may represent

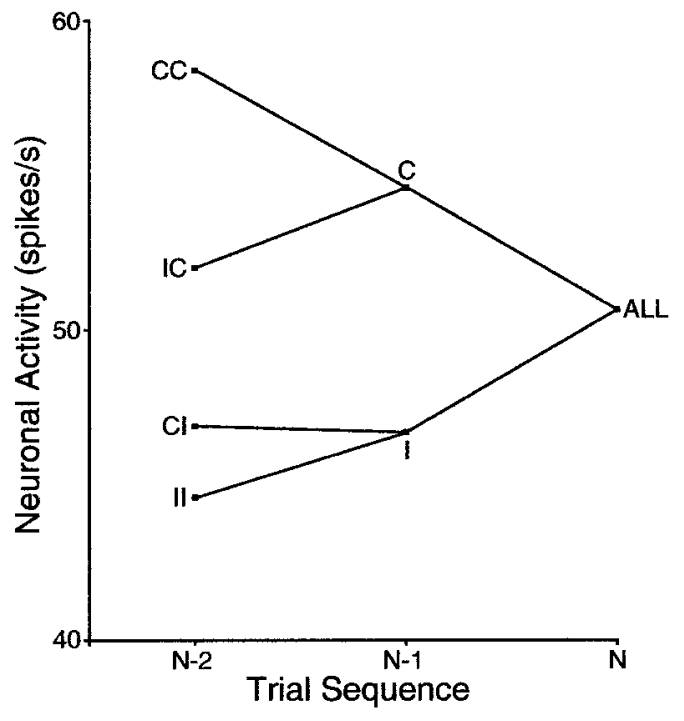

Figure 4. Distribution of pretarget neuronal activity as a function of the sequence of previous saccades. We limited our analysis to those neurons with at least five trials in each condition $(n=35)$. Because a block consisted of between 50 and 100 trials and because even a low error rate dramatically decreased our yield using this analysis, we could reliably look back only to trial history $\mathrm{N}-2$. The current mean neuronal activity is shown irrespective of the location of the previous saccades $(N)$ or based on the compatibility of the previous saccade $(N-1)$ or previous two saccades $(N-2)$ with the response field of each neuron. The compatibility of recent to distant previous saccades is determined by reading from right to left with the metrics of each saccade being either compatible $(C)$ or incompatible $(I)$ with respect to the response field of the neuron. 
a form of neural plasticity that is required to interact with environmental conditions and achieve behavioral goals that are dynamic. Certainly the transitory changes observed here are not caused by changes in connectivity or morphological changes at the cellular and synaptic levels associated with more traditional views of plasticity. The observed neural plasticity is likely caused by afferent modulatory input that uses an otherwise unchanging connectivity as envisioned by Mountcastle (1995).

The basis of this putative neural plasticity is, as yet, unknown. The intermediate layers of the SC receive input from a variety of cortical and subcortical areas (Sparks and Hartwich-Young, 1989), and the activity of SC neurons can be influenced by cognitive strategies that involve visual attention, expectation of the next target or movement, and movement preparation (Glimcher and Sparks, 1992; Kustov and Robinson, 1996; Dorris and Munoz, 1998). It is therefore plausible that the SC neuronal plasticity could be produced by such cognitive influences (Kirby, 1976; Squires et al., 1976) and that the monkey imparted more weight to the most recent target location as it calculated the likelihood of the next movement, although this strategy is probabilistically unjustified. Conversely, these sequential patterns may be caused by automatic changes in the response bias of the motor system (Link, 1992) that may be fundamental to the enduring changes in movement parameters that result from long-term training involving movement repetition. This latter view is supported by our previous studies using the same gap saccade paradigm. Short-term exposure to the repeated presentation of an identical saccadic target (dozens of repeated compatibles over minutes) resulted in an adaptive reduction in SRTs with covarying changes in neuronal activity (Dorris and Munoz, 1998). Furthermore, extended training in this paradigm (thousands of repeated compatibles over days) resulted in enduring reductions in SRTs restricted to the saccades made to the trained target location (Paré and Munoz, 1996). This time-dependent progression in performance improvement suggests that the immediate neural plasticity observed here may represent the initial building block in the process of motor learning. The analysis of sequential patterns of both behavior and neuronal activities used in this study may prove to be a powerful tool in tracking the development of the fleeting form of motor plasticity observed here into the enduring changes that occur with directed training.

\section{REFERENCES}

Anderson JR (1980) Cognitive skills and their acquisition. Hillsdale, NJ: Erlbaum

Bertelson P (1961) Sequential redundancy and speed in a serial twochoice responding task. Q J Exp Psychol 13:90-120.

Buonomano DV, Merzenich MM (1998) Cortical plasticity: from synapses to maps. Annu Rev Neurosci 21:149-186.

Carpenter RHS (1981) Oculomotor Procrastination. In: Eye movements: cognition and visual perception (Fisher DF, Monty RA, Senders JW, eds), pp 237-246. Hillsdale, NJ: Erlbaum.

Classen J, Liepert J, Wise SP, Hallett M, Cohen LG (1998) Rapid plasticity of human cortical movement representation induced by practice. J Neurophysiol 79:1117-1123.

Dias EC, Bruce CJ (1994) Physiological correlate of fixation disengagement in the primate's frontal eye field. J Neurophysiol 72:2532-2537.

Dorris MC, Munoz DP (1995) A neural correlate for the gap effect on saccadic reaction times in monkey. J Neurophysiol 95:2558-2562.
Dorris MC, Munoz DP (1998) Saccadic probability influences motor preparation signals and time to saccadic initiation. $\mathrm{J}$ Neuosci 98:7015-7026.

Dorris MC, Paré M, Munoz DP (1997) Neuronal activity in monkey superior colliculus related to the initiation of saccadic eye movements. J Neuosci 97:8566-8579.

Fischer B, Ramsperger E (1986) Human express-saccades: effects of randomization and daily practice. Exp Brain Res 64:569-578.

Glimcher PW, Sparks DL (1992) Movement selection in advance of action in the superior colliculus. Nature 92:542-545.

Hanes DP, Schall JD (1996) Neural control of voluntary movement initiation. Science 274:427-430.

Hanes DP, Thompson KG, Schall JD (1995) Relationship of presaccadic activity in frontal eye field and supplementary eye field to saccade initiation in macaque: Poisson spike train analysis. Exp Brain Res 103:85-96.

Jüttner M, Wolf W (1992) Occurence of human express saccades depends on stimulus uncertainty and stimulus sequence. Exp Brain Res 89:678-681.

Karni A, Meyer G, Jezzard P, Adams MM, Turner R, Ungerleider LG (1995) Functional MRI evidence for adult motor cortex plasticity during motor skill learning. Nature 377:155-158.

Kirby NH (1976) Sequential effects in two-choice reaction time: Automatic facilitation or subjective expectancy? J Exp Psychol Hum Percept Perform 2: 567-577.

Kustov AA, Robinson DL (1996) Shared neural control of attentional shifts and eye movements. Nature 384:74-77.

Link SW (1992) The wave theory of difference and similarity (Scientific Psychology Series). Hillsdale, NJ: Erlbaum.

McLaughlin SC (1967) Parametric adjustment in saccadic eye movements. Percept Psychophys 2:359-362.

Miles FA, Eighmy BB (1980) Long-term adaptive changes in primate vestibuloocular reflex. I. Behavioral observations. J Neurophysiol 80:1406-1425.

Miles FA, Lisberger SG (1981) Plasticity in the vestibulo-ocular reflex: a new hypothesis. Annu Rev Neurosci 81:4:273-299.

Mitz AR, Godschalk M, Wise SP (1991) Learning-dependent neuronal activity in the premotor cortex: activity during the acquisition of conditional motor associations. J Neurosci 11:1855-1872.

Mountcastle VB (1995) The parietal system and some higher brain functions. Cereb Cortex 5:377-390.

Nakamura K, Sakai K, Hikosaka O (1998) Neuronal activity in medial frontal cortex during learning of sequential procedures. J Neurophysiol 80:2671-87.

Nudo RJ, Milliken GW, Jenkins WM, Merzenich MM (1996) Usedependent alterations of movement representations in primary motor cortex of adult squirrel monkeys. J Neuosci 96:785-807.

Paré M, Munoz DP (1996) Saccadic reaction time in the monkey: advanced preparation of oculomotor programs is primarily responsible for express saccade occurrence. J Neurophysiol 76:3666-3681.

Pascual-Leone A, Grafman J, Hallett M (1994) Modulation of cortical motor output maps during development of implicit and explicit knowledge. Science 263:1287-1289.

Remington RJ (1969) Analysis of sequential effects on choice reaction times. J Exp Psychol 82:250-257.

Ross LE, Ross SM (1980) Saccade latency and warning signals: stimulus onset, offset, and change as warning events. Percept Psychophys 27:251-257.

Schmidt RA (1991) Motor skill acquisition. In: Encyclopedia of human biology (Dulbecco R, ed), pp 121-129. Orlando, FL: Academic.

Soetens E (1998) Localizing sequential effects in serial choice reaction time with the information reduction procedure. J Exp Psychol Hum Percept Perform 24:547-568.

Sparks DL, Hartwich-Young R (1989) The deep layers of the superior colliculus. Rev Oculomotor Res 89:3:213-255.

Squires KC, Wickens C, Squires NK, Donchin E (1976) The effect of stimulus sequence on the waveform of the cortical event-related potential. Science 193:1142-1146. 